\title{
Biotribological properties of xerostomia patient saliva and its enhancement
}

\author{
Prashant K. Sharma ${ }^{1 *}$, Jeroen Vinke ${ }^{1}$, Marijn Oude Elberink ${ }^{1}$, Monique A. Stokman ${ }^{2}$, Frans G.M. Kroese ${ }^{3}$, Kamran \\ $\mathrm{Nazmi}^{4}$, Floris J. Bikker ${ }^{4}$, Henny C. van der $\mathrm{Mei}^{1}$, Arjan Vissink ${ }^{5}$ \\ ${ }^{1}$ University of Groningen and University Medical Center Groningen, Department of Biomedical Engineering, Groningen, The \\ Netherlands \\ ${ }^{2}$ University of Groningen and University Medical Center Groningen, Department of Radiation Oncology, Groningen, The \\ Netherlands \\ ${ }^{3}$ University of Groningen and University Medical Center Groningen, Department of Rheumatology and Clinical Immunology, \\ Groningen, The Netherlands \\ ${ }^{4}$ Academic Centre for Dentistry Amsterdam, Free University and University of Amsterdam, Department of Oral Biochemistry, \\ Amsterdam, The Netherlands \\ ${ }^{5}$ University of Groningen and University Medical Center Groningen, Department of Oral and Maxillofacial Surgery, Groningen, The \\ Netherlands
}

\begin{abstract}
Objectives The study aimed to quantify the lubricating properties of chewing stimulated whole saliva from healthy controls $(n=22)$, from patients suffering from primary Sjögren's syndrome $(n=37)$ and from patients undergoing head-and-neck radiotherapy $(\mathrm{n}=34)$. Materials and Methods All participants had to complete the Xerostomia Inventory questionnaire to score dry mouth sensation. Lubrication was measured using an ex vivo tongue-enamel friction system in terms of Relief and Relief period. MUC5b and total protein concentrations of the saliva samples were measured by an enzyme-linked immunosorbent assay and a bicinchoninic acid assay, respectively. Results Relief of Sjögren's patients saliva and post-irradiation patients saliva was similar compared with healthy controls, but saliva from post-irradiation patients lubricated significantly better than saliva from Sjögren's patients. The Relief period was similar between the three groups. The Relief and Relief period were higher for saliva samples post-irradiation compared to pre-irradiation. MUC5b and total protein concentrations were comparable in all groups. MUC5b and total protein output were significantly lower in patients subjected to radiotherapy compared to saliva from healthy controls and pre-irradiation patients. MUC5b concentrations positively correlated with lubricating properties of post-irradiation patient saliva. Conclusions The lubricating properties of patient saliva were not any worse than healthy controls. Lower flow rate leads to lower availability of saliva in the oral cavity and decreases the overall output of protein and MUC5b, which might result in an insufficient replenishing of the mucosal salivary film. Clinical Relevance An insufficient replenishing might underlie the sensation of a dry mouth and loss of oral function. In the talk I will explain biomaterials related strategies, yet ex vivo, to enhance salivary lubrication despite of low flowrates.
\end{abstract}

\footnotetext{
${ }^{*}$ Corresponding author: p.k.sharma@umcg.nl
} 
(a) Salivary flow rate

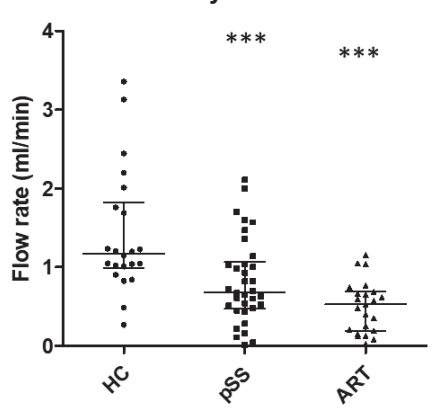

(d)

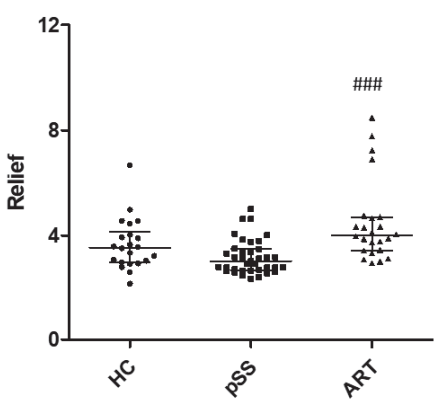

(g)

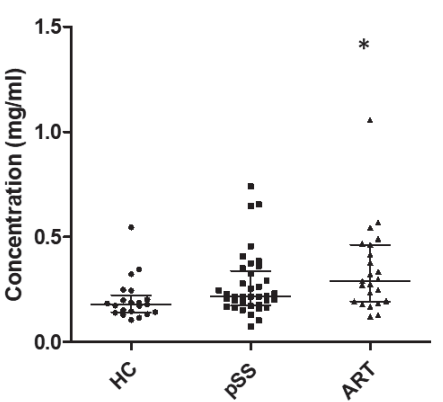

(b)

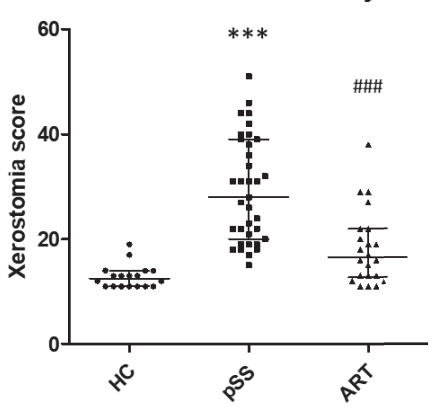

(e)

Relief period

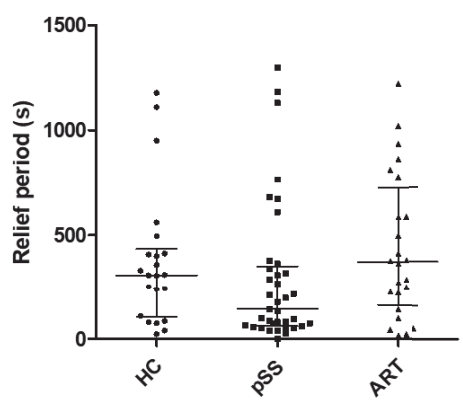

(h)

MUC5b output

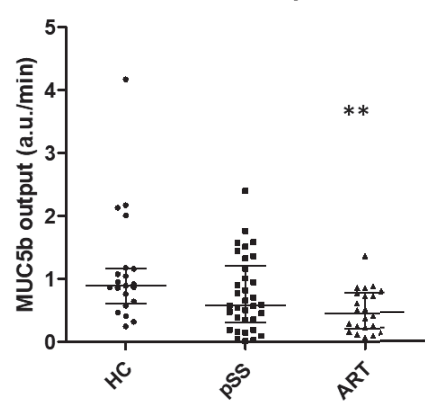

(c) Relief $_{\max }$

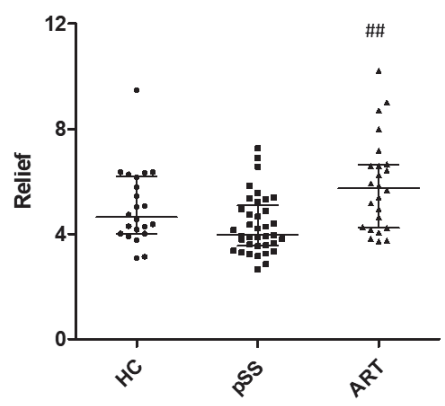

(f)

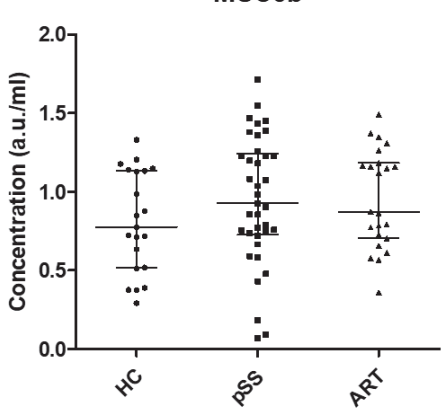

(i)

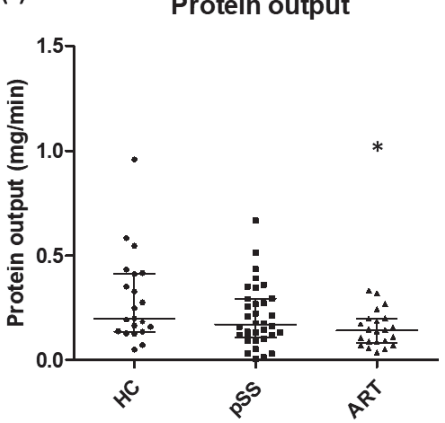

Fig. 1 Differences in saliva characteristics and dry mouth sensation of healthy controls (HC), primary Sjögren's syndrome patients (pSS) and head-and neck radiotherapy patients after radiotherapy (ART). (a) Flow rate of stimulated whole saliva. (b) Xerostomia Inventory

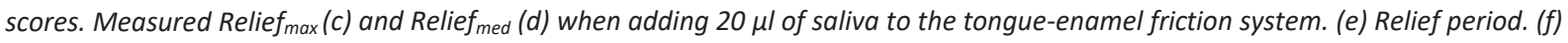
MUC5b concentration. (g) Protein concentration. (h) MUC5b output. (i) Protein output. Statistical differences compared to HC are marked by * $(p<0.05), * *(p<0.01)$ or *** $(p<0.001)$. \# indicates significant differences between ART and pSS. Error bars represent the interquartile ranges and median value 